\title{
MAXIMAL TORI IN THE CONTACTOMORPHISM GROUPS OF CIRCLE BUNDLES OVER HIRZEBRUCH SURFACES
}

\author{
EUGENE LERMAN
}

\begin{abstract}
In a recent preprint Yael Karshon showed that there exist nonconjugate tori in a group of symplectomorphisms of a Hirzebruch surface. She counted them in terms of the cohomology class of the symplectic structure. We show that a similar phenomenon exists in the contactomorphism groups of prequantum circle bundles over Hirzebruch surfaces. Note that the contact structures in question are fillable. This may be contrasted with an earlier paper where we showed that there are infinitely many non-conjugate tori in the contactomorphism groups of certain overtwisted lens spaces (Contact cuts, Israel J. Math. 124 (2001), $77-92)$.
\end{abstract}

\section{Introduction}

Consider the product of two projective spaces $M=\mathbb{C} P^{1} \times \mathbb{C} P^{1}$. Let $\omega_{1}$ and $\omega_{2}$ denote the pull-backs of the standard area form on $\mathbb{C} P^{1}=S^{2}$ by the two projection maps (so that $\int_{\mathbb{C} P^{1} \times\{*\}} \omega_{1}=1$ etc.). Let $\omega_{a, b}=a \omega_{1}+b \omega_{2}$; it is a symplectic form if $a, b>0$. Gromov in his fundamental paper [G] showed that the topology of the group of symplectomorphisms of $\left(M, \omega_{a, b}\right)$ changes as the ratio $a / b$ crosses integers. The rational cohomology ring of the identity component of the group of symplectomorphisms of $\left(M, \omega_{a, b}\right)$ has been computed for different values of $a / b$ by Abreu [Ab] and Abreu - McDuff [AbMc]. Karshon $[\mathrm{K}]$ showed that for $a \geq b>0$ the number of conjugacy classes of maximal tori in the group of Hamiltonian symplectomorphism of $\left(M, \omega_{a, b}\right)$ is the number of integers $k$ satisfying $0 \leq k<a / b$. Similar results hold for a one point blow-up $\widetilde{\mathbb{C} P^{2}}$ of $\mathbb{C} P^{2}$.

Now suppose that $a$ and $b$ are positive integers. Then the symplectic form $\omega_{a, b}$ is integral and so the Boothby-Wang construction [BW] produces a contact form $A_{a, b}$ on the principal circle bundle $P=P(a, b)$ over $M$ with Chern class $c_{1}(P)=\left[\omega_{a, b}\right]$. We will show in Theorem 3.1 that for $a \geq b>0$ the number of conjugacy classes of maximal tori in the group of contactomorphisms of $P(a, b)$ is at least the number of integers $k$ satisfying $0 \leq k<a / b$. Similar results hold for circle bundles over $\widetilde{\mathbb{C} P^{2}}$. Since all contact manifolds $\left(P(a, b), A_{a, b}\right)$ can be

Received July 17, 2002.

The author was supported by NSF grant DMS-980305 and by R. Kantorovitz. 
given the structure of a $K$-contact manifold this answers positively Problem 3 in [Y1]. In fact the manifolds $P(a, b)$ are Sasakian. ${ }^{1}$

\section{Preliminaries}

In this section we review contact forms, contact structures, the Boothby-Wang construction, symplectization, symplectic cones, contact group actions, contact moment maps, contact and symplectic toric manifolds.

Definition 2.1. Recall that a 1-form $\alpha$ on a manifold $P$ is contact if $\alpha_{x} \neq 0$ for any $x \in P$, so $\xi=\operatorname{ker} \alpha$ is a codimension- 1 distribution, and if additionally $\left.d \alpha\right|_{\xi}$ is non-degenerate. Thus the vector bundle $\xi \rightarrow P$ necessarily has even-dimensional fibers, and the manifold $M$ is necessarily odd-dimensional. A codimension-1 distribution $\xi$ on a manifold $P$ is a (co-oriented) contact structure if it is the kernel of a contact form.

Throughout the paper $\alpha$ will always denote a contact form and $\xi$ will always denote a contact structure. We will refer either to a pair $(P, \alpha)$ or $(P, \xi)$ as a contact manifold.

It is a standard fact that $\xi \subset T P$ is contact iff the punctured line bundle $\xi^{\circ} \backslash P$ is a symplectic submanifold of the cotangent bundle $T^{*} P$. Here $\xi^{\circ}$ denotes the annihilator of $\xi$ in $T^{*} P$. Now suppose $\xi=\operatorname{ker} \alpha$ for some contact form $\alpha$. Then $\alpha$, thought of as a map from $P$ to $T^{*} P$ is a global nowhere zero section of $\xi^{\circ}$. Hence $\xi^{\circ} \backslash P$ has two components. Denote one of them by $\xi_{+}^{\circ}$.

Definition 2.2. A symplectic manifold $(M, \omega)$ is a symplectic cone if there exists a free proper action $\left\{\rho_{t}\right\}$ of the real line on $M$ such that $\rho_{t}^{*} \omega=e^{t} \omega$ for all $t \in \mathbb{R}$. The vector field $X$ generating $\left\{\rho_{t}\right\}$ then satisfies $L_{X} \omega=\omega$. We will refer to $X$ as the Liouville vector field. Note that $d \iota(X) \omega=\omega$.

We recall that if $(M, \omega)$ is a symplectic cone, then the orbit space $M / \mathbb{R}$ for the action of the real line is naturally a contact manifold. We will refer to the contact manifold $M / \mathbb{R}$ as the base of the symplectic cone $(M, \omega)$.

Conversely, if $(P, \xi)$ is a contact manifold then $\xi_{+}^{\circ} \subset T^{*} P$ is a symplectic cone under the action of $\mathbb{R}$ given by $\rho_{t}(p, \eta)=\left(p, e^{t} \eta\right)$ for all $p \in P, \eta \in\left(\xi_{+}^{\circ}\right)_{p} \subset T_{p}^{*} P$. We will refer to $\xi_{+}^{\circ}$ as a symplectization of $(P, \xi)$. If $(M, \omega)$ is a symplectic cone with the base $(P, \xi)$ then it is not hard to show that $(M, \omega)$ is symplectomorphic to $\xi_{+}^{\circ}$ and that moreover the symplectomorphism is equivariant with respect to the two $\mathbb{R}$ actions.

If $(M, \omega)$ is a symplectic cone with a Liouville vector field $X$ and if a Lie group $G$ acts on $M$ preserving $\omega$ and $X$ then $G$ preserves $\iota(X) \omega$. Hence $\Phi: M \rightarrow \mathfrak{g}^{*}$ given by

$$
\langle\Phi, Y\rangle=\iota\left(Y_{M}\right) \iota(X) \omega
$$

\footnotetext{
${ }^{1}$ They also carry Einstein metrics [WZ], but the Sasakian and the Einstein metrics are not the same unless $a=b$; cf. [BG, Theorem 2.4 (iv)].
} 
for all $Y \in \mathfrak{g}$ is a moment map. Here $Y_{M}$ denotes a vector field induced by $Y$ on $M: Y_{M}(x)=\left.\frac{d}{d t}\right|_{t=0}(\exp t Y) \cdot x$. Note that $\Phi\left(\rho_{t}(m)\right)=e^{t} \Phi(m)$ for all $t \in \mathbb{R}$, $m \in M$.

If $(M, \omega)$ is a symplectic manifold and the cohomology class $[\omega] \in H^{2}(M, \mathbb{R})$ is integral then there exists a principal circle bundle $\pi: P \rightarrow M$ with first Chern class $c_{1}(P)=[\omega]$. A theorem of Boothby and Wang asserts that moreover there exists a connection 1-form $A$ on $P$ with $d A=\pi^{*} \omega$ and that consequently $A$ is a contact 1-form [BW, Theorem 3]. We will refer to the contact manifold $(P, \xi=\operatorname{ker} A)$ as the Boothby-Wang manifold of $(M, \omega)$.

An action of a Lie group $G$ on a contact manifold $(P, \xi)$ is contact if it preserves the contact distribution $\xi$. If furthermore $G$ is compact and connected then there exists a $G$-invariant contact form $\alpha$ with $\operatorname{ker} \alpha=\xi$ (take any contact form $\alpha_{0}$ with ker $\alpha_{0}=\xi$ and average it over $G$ ).

Suppose now that a Lie group $G$ acts on a manifold $P$ preserving a contact form $\alpha$. We define the corresponding $\alpha$-moment map $\Psi_{\alpha}: P \rightarrow \mathfrak{g}^{*}$ by

$$
\left\langle\Psi_{\alpha}(x), X\right\rangle=\alpha_{x}\left(X_{P}(x)\right)
$$

for all $x \in P$ and all $X \in \mathfrak{g}$, where as above $X_{P}$ denotes the vector field induced by $X$ on $P$. One can show that $\left(P, \alpha, \Psi_{\alpha}: P \rightarrow \mathfrak{g}^{*}\right)$ completely encodes the (infinitesimal) action of $G$ on $P$.

Note that if $f$ is a $G$-invariant nowhere zero function, then $\alpha^{\prime}=f \alpha$ is also a $G$-invariant contact form defining the same contact structure. Clearly the corresponding moment map $\Psi_{\alpha^{\prime}}$ satisfies $\Psi_{\alpha^{\prime}}=f \Psi_{\alpha}$. Thus the definition of a contact moment map above is somewhat problematic: it depends on a choice of an invariant contact form rather then solely on the contact structure and the action. Fortunately there is also a notion of a contact moment map that doesn't have this problem. Namely, suppose again that a Lie group $G$ acts on a manifold $P$ preserving a contact structure $\xi$ (and its co-orientation). The lift of the action of $G$ to the cotangent bundle then preserves a component $\xi_{+}^{\circ}$ of $\xi^{\circ} \backslash P$. The restriction $\Psi=\left.\Phi\right|_{\xi_{+}^{\circ}}$ of the moment map $\Phi$ for the action of $G$ on $T^{*} P$ to depends only on the action of the group and on the contact structure. Moreover, since $\Phi: T^{*} P \rightarrow \mathfrak{g}^{*}$ is given by the formula

$$
\langle\Phi(p, \eta), X\rangle=\left\langle\eta, X_{P}(p)\right\rangle
$$

for all $p \in P, \eta \in T_{p}^{*} P$ and $X \in \mathfrak{g}$, we see that if $\alpha$ is any invariant contact form with $\operatorname{ker} \alpha=\xi$ then $\left\langle\alpha^{*} \Psi(p), X\right\rangle=\left\langle\alpha^{*} \Phi(p), X\right\rangle=\left\langle\alpha_{p}, X_{P}(p)\right\rangle=\left\langle\Psi_{\alpha}(p), X\right\rangle$. Here we think of $\alpha$ as a section of $\xi_{+}^{\circ} \rightarrow M$. Thus $\Psi \circ \alpha=\Psi_{\alpha}$, that is, $\Psi=\left.\Phi\right|_{\xi_{+}^{\circ}}$ is a "universal" moment map.

Definition 2.3. Let $(P, \xi)$ be a co-oriented contact manifold with an action of a Lie group $G$ preserving the contact structure $\xi$ and its co-orientation. Let $\Psi: \xi_{+}^{\circ} \rightarrow \mathfrak{g}^{*}$ denote the corresponding moment map. We define the moment cone $C(\Psi)$ to be the set

$$
C(\Psi):=\Psi\left(\xi_{+}^{\circ}\right) \cup\{0\}
$$


If $\alpha$ is a $G$-invariant contact form with $\xi=\operatorname{ker} \alpha$ and $\alpha(P) \subset \xi_{+}^{\circ}$, then

$$
C(\Psi)=\left\{t \Psi_{\alpha}(p) \mid p \in P, t \in[0, \infty)\right\}=\mathbb{R}^{\geq 0} \Psi_{\alpha}(P),
$$

where $\Psi_{\alpha}: M \rightarrow \mathfrak{g}^{*}$ denotes the $\alpha$-moment map. The moment cone, unlike the $\alpha$-moment map or its image, is an invariant of the contact group action.

Recall that a symplectic toric $G$-manifold is a triple $\left(M, \omega, \Phi: M \rightarrow \mathfrak{g}^{*}\right)$ where $M$ is manifold with an effective Hamiltonian action of a torus $G$ preserving the symplectic form $\omega$ and satisfying $\operatorname{dim} M=2 \operatorname{dim} G$, and $\Phi$ is a corresponding moment map.

For this and other reasons we say that an effective contact action of a torus $G$ on a contact manifold $(P, \xi=\operatorname{ker} \alpha)$ is toric if the induced action of $G$ on the symplectization $\xi_{+}^{\circ}$ of $P$ makes it a symplectic toric manifold. Thus a contact toric $G$-manifold is a triple $\left(P, \xi=\operatorname{ker} \alpha, \Psi_{\alpha}: P \rightarrow \mathfrak{g}^{*}\right)$ where $P$ is a manifold with an effective action of a torus $G$ on $P$ preserving a contact form $\alpha$ and satisfying $\operatorname{dim} P+1=2 \operatorname{dim} G$, and $\Psi_{\alpha}: P \rightarrow \mathfrak{g}^{*}$ is the corresponding $\alpha$-moment map.

Remark 2.4. If a torus $G$ acts effectively and contactly on a contact manifold $(P, \xi)$ then $\operatorname{dim} G \leq \frac{1}{2}(\operatorname{dim} P+1)$ (see, for instance, $\left.[\mathrm{LS}]\right)$. Thus if $(P, \xi=$ ker $\left.\alpha, \Psi_{\alpha}: P \rightarrow \mathfrak{g}^{*}\right)$ is a contact toric manifold then $G$ is a maximal torus in the group of contact diffeomorphisms $\operatorname{Diff}(P, \xi)$.

\section{Main theorem}

We are now ready to state the main result of the paper.

Theorem 3.1. Let $M$ denote a Hirzerbruch surface (that is, $M$ is diffeomorphic to $S^{2} \times S^{2}$ or to $\widetilde{\mathbb{C} P^{2}}$ ). For every integer $\ell>1$ there exists a principal circle bundle $P$ over $M$ and a contact structure $\xi$ on $P$ such that the group of contactomorphisms

$$
\operatorname{Diff}(P, \xi)=\{\varphi \in \operatorname{Diff}(P) \mid d \varphi(\xi)=\xi\}
$$

has at least $\ell$ non-conjugate maximal tori.

Remark 3.2. It was shown in [L3] that every lens space $L(p, q)$ has a contact structure $\xi$ such that the group $\operatorname{Diff}(L(p, q), \xi)$ of contactomorphisms has infinitely many non-conjugate maximal tori. However, these contact structures were all overtwisted and it was their "flexibility" that made the construction work. In Theorem 3.1 above the contact structures are fillable.

We now begin our proof of Theorem 3.1. First let us spell out exactly what we mean by two contact toric manifolds being isomorphic.

Definition 3.3. We say that two contact toric $H$-manifolds $\left(P_{i}, \xi_{i}=\operatorname{ker} \alpha_{i}, \Psi_{\alpha_{i}}\right.$ : $\left.P_{i} \rightarrow \mathfrak{h}^{*}\right), i=1,2$ are isomorphic if there exists a diffeomorphism $\varphi: P_{1} \rightarrow P_{2}$ with $d \varphi\left(\xi_{1}\right)=\xi_{2}$ and an isomorphism $\gamma: H \rightarrow H$ such that

$$
\varphi(g \cdot p)=\gamma(g) \cdot \varphi(p)
$$


for all $g \in H$, all $p \in P_{1}$.

Remark 3.4. If $\left(P_{i}, \xi_{i}=\operatorname{ker} \alpha_{i}, \Psi_{\alpha_{i}}: P_{i} \rightarrow \mathfrak{h}^{*}\right), i=1,2, \varphi: P_{1} \rightarrow P_{2}$ and $\gamma: H \rightarrow H$ are as above then

$$
\Psi_{\alpha_{2}} \circ \varphi= \pm T \circ\left(e^{f} \Psi_{\alpha_{1}}\right)
$$

for some function $f \in C^{\infty}\left(P_{1}\right)$. Here $T=\left(d \gamma^{-1}\right)^{*}: \mathfrak{h}^{*} \rightarrow \mathfrak{h}^{*}$ (the sign in (3.1) is + if the contactomorphism $\varphi$ preserves the co-orientations of the contact structures). Hence $\pm T$ maps the moment cone $C\left(\Psi_{1}\right)$ isomorphically onto the moment cone $C\left(\Psi_{2}\right)$. Also, the linear map $T$ preserves the weight lattice $\mathbb{Z}_{H}^{*}$ of $H$, hence $T \in \mathrm{GL}\left(\mathbb{Z}_{H}^{*}\right)$. Therefore in order to prove that two contact toric $H$-manifolds are not isomorphic it is enough prove that there does not exist $T \in \mathrm{GL}\left(Z_{H}^{*}\right)$ mapping one moment cone onto another.

Next let me try to explain what makes Theorem 3.1 true. As mentioned in the introduction Yael Karshon constructed for every integer $\ell>1$ collections $\left\{\left(M_{i}, \omega_{i}, \Phi_{i}: M_{i} \rightarrow \mathfrak{g}^{*}\right)\right\}_{i=1}^{\ell}$ of symplectic toric 4-manifolds (diffeomorphic to $S^{2} \times S^{2}$ or to $\widetilde{\mathbb{C} P^{2}}$ ) which are pairwise non-isomorphic as symplectic toric manifolds but are isomorphic as symplectic manifolds [K] (the group $G$ is a 2-torus). As we recalled above, by a theorem of Boothby and Wang for every integral symplectic manifold $(M, \omega)$ there exists a principal circle bundle $\pi: P \rightarrow M$ with a connection 1-form $A$ such that $d A=\pi^{*} \omega$. Clearly if two integral symplectic manifolds are symplectomorphic the corresponding Boothby-Wang manifolds are contactomorphic. Therefore our proof of Theorem 3.1 amounts to: choose a Karshon collection of integral symplectic toric $G$-manifolds $M_{i}$. The corresponding Boothby-Wang manifolds $P_{i}$ are all contactomorphic. The actions of $G$ on the $M_{i}$ are covered by actions of extensions of $G$ by $S^{1}$ on $P_{i}$. These extensions are simply $G \times S^{1}$. Moreover since the $M_{i}$ 's are not isomorphic as symplectic $G$-manifolds, the $P_{i}$ 's should be non-isomorphic as contact $G \times S^{1}$ manifolds. We should be able to ascertain this by looking at their moment cones. However, I found it simpler to proceed a little differently. Namely given the moment polytopes of the $M_{i}$ 's we will guess what the moment cones of the $P_{i}$ 's should be and then prove that (a) the guess is correct and (b) there are no elements of $\mathrm{GL}\left(\mathbb{Z}_{G}^{*} \times \mathbb{Z}^{*}\right)$ mapping one moment cone into another.

Remark 3.5. By a theorem of Banyaga the group $\operatorname{Diff}(P, A)$ of diffeomorphisms of $P$ preserving the connection $A$ is an extension by $S^{1}$ of the group of symplectomorphisms $\operatorname{Diff}(M, \omega)$. However the group $\operatorname{Diff}(P, \xi)$ is much bigger than the group $\operatorname{Diff}(P, A)$. Thus we have to do work in order to show that the tori in $\operatorname{Diff}(P, \xi)$ that we got by lifting non-conjugate tori in $\operatorname{Diff}(M, \omega)$ to $\operatorname{Diff}(P, A)$ are in fact non-conjugate in $\operatorname{Diff}(P, \xi)$.

3.1. Boothby-Wang construction for symplectic toric manifolds. As above we let $\mathfrak{g}^{*}$ denote the vector space dual of the Lie algebra of a torus $G$, and $\mathbb{Z}_{G}^{*}$ denote the weight lattice of $G$. A polytope $\Delta \subset \mathfrak{g}^{*}$ is Delzant if for any vertex $v^{*}$ of $\Delta$ there exists a basis $\left\{u_{i}^{*}\right\}$ of $\mathbb{Z}_{G}^{*}$ such that every edge of $\Delta$ coming 
out of $v^{*}$ is of the form $\left\{v^{*}+t u_{i}^{*} \mid 0 \leq t \leq a_{i}\right\}$ for some $a_{i} \geq 0$. In particular $\Delta$ is simple and rational. Recall

Theorem 3.6 (Delzant [D]). If $\mathfrak{g}^{*}$ the dual of the Lie algebra of a torus $G$ and $\Delta \subset \mathfrak{g}^{*}$ is a Delzant polytope then there exists a unique (compact connected) symplectic toric $G$-manifold $\left(M_{\Delta}, \omega_{\Delta}, \Phi_{\Delta}: M_{\Delta} \rightarrow \mathfrak{g}^{*}\right)$ with $\Phi_{\Delta}\left(M_{\Delta}\right)=\Delta$.

Conversely if $\left(M, \omega, \Phi: M \rightarrow \mathfrak{g}^{*}\right)$ is a (compact connected) symplectic toric manifold then the moment polytope $\Phi(M)$ is a Delzant polytope.

We next recall a few well-known facts about (symplectic) toric manifolds (cf. $[\mathrm{DJ}])$. Let $\left(M_{\Delta}, \omega_{\Delta}, \Phi: M_{\Delta} \rightarrow \mathfrak{g}^{*}\right)$ be a symplectic toric manifold with moment polytope $\Delta$. Then

1. The integral cohomology $H^{*}\left(M_{\Delta}, \mathbb{Z}\right)$ is generated by degree 2 classes.

2. $H_{2}\left(M_{\Delta}, \mathbb{Z}\right)$ is generated by the preimages $\Phi^{-1}(e)$ where $e$ 's are the edges of $\Delta$. Each preimage is a 2 -sphere, and this set of generators is redundant.

3. If $e=\left\{v^{*}+t u^{*} \mid 0 \leq t \leq a\right\}$ is an edge of $\Delta$ and $u^{*} \in \mathbb{Z}_{G}^{*}$ is primitive, then $\left\langle\left[\omega_{\Delta}\right], \Phi^{-1}(e)\right\rangle=a$.

Thus the symplectic form $\omega_{\Delta}$ is integral iff the edges of $\Delta$ can be represented by elements of $\mathbb{Z}_{G}^{*}$. Hence if one vertex of $\Delta$ lies in the weight lattice (and $\omega_{\Delta}$ is integral) then all vertices of $\Delta$ lie in the weight lattice. Therefore it is no loss of generality to assume that if $\left(M, \omega, \Phi: M \rightarrow \mathfrak{g}^{*}\right)$ is a (compact connected) integral symplectic toric $G$-manifold then the moment polytope $\Phi(M)$ is an integral polytope, i.e., that all of its vertices are in the integral lattice $\mathbb{Z}_{G}^{*}$.

I showed in [L2] that an analogue of Theorem 3.6 holds for symplectic cones. Let us recall the result. Let $\mathfrak{h}^{*}$ denote the dual of the Lie algebra of a torus $H, \mathbb{Z}_{H}^{*} \subset \mathfrak{h}^{*}$ denote its weight lattice and $\mathbb{Z}_{H} \subset \mathfrak{h}$ denote the integral lattice. A cone $C \subset \mathfrak{h}^{*}$ is good if there exists a (nonempty) set of primitive vectors $\mu_{1}, \ldots, \mu_{N} \in \mathbb{Z}_{H}$ such that

1.

$$
C=\bigcap_{j}\left\{\eta \in \mathfrak{h}^{*} \mid\left\langle\eta, \mu_{j}\right\rangle \geq 0\right\}
$$

2. The set $\left\{\mu_{1}, \ldots, \mu_{N}\right\}$ is minimal, i.e.,

$$
C \neq \bigcap_{j \neq i}\left\{\eta \in \mathfrak{h}^{*} \mid\left\langle\eta, \mu_{j}\right\rangle \geq 0\right\}
$$

for any $i, 0<i \leq N$.

3. Any codimension $k$ face $F$ of $C(k \neq \operatorname{dim} G)$ is the intersection of exactly $k$ facets, and the normals to these facets generate a direct summand of rank $k$ of the integral lattice $\mathbb{Z}_{H}$.

For example, if $\Delta \subset \mathfrak{g}^{*}$ is an integral Delzant polytope then

$$
C_{\Delta}:=\left\{t\left(\eta, 1^{*}\right) \in \mathfrak{g}^{*} \times \mathbb{R}^{*} \mid \eta \in \Delta, t \geq 0\right\}
$$

is a good cone in $\mathfrak{h}^{*}=\mathfrak{g}^{*} \times \mathbb{R}^{*}$. Here $\left\{1^{*}\right\}$ is a basis of $\mathbb{Z}^{*}$ dual to the basis $\{1\}$ of $\mathbb{Z}$, and we think of $\mathfrak{h}^{*}$ as the dual of the Lie algebra of $H=G \times \mathbb{R} / \mathbb{Z}$. We will refer to $C_{\Delta}$ as the standard cone on the polytope $\Delta$. 
Lemma 3.7. Let $\mathfrak{h}^{*}$ denote the dual of the Lie algebra of a torus $H$. Given a good cone $C \subset \mathfrak{h}^{*}$ there exists a unique (compact connected) contact toric manifold $\left(P_{C}, \xi_{C}=\operatorname{ker} \alpha_{C}, \Psi_{\alpha_{C}}: P_{C} \rightarrow \mathfrak{h}^{*}\right)$ with moment cone $C$.

Equivalently there exists a unique symplectic toric cone (with a compact connected base) $\left(M_{C}, \omega_{C}, \Phi_{C}: M_{C} \rightarrow \mathfrak{h}^{*}\right)$ such that $\Phi_{C}\left(M_{C}\right)=C \backslash\{0\}$. Additionally the fibers of $\Phi_{C}$ are connected.

Proof. See Theorem 2.8 in [L2].

Lemma 3.8. Let $\mathfrak{g}^{*}$ denote the dual of the Lie algebra of a torus $G, \Delta \subset \mathfrak{g}^{*}$ be an integral Delzant polytope, $C=C_{\Delta} \subset \mathfrak{g}^{*} \times \mathbb{R}^{*}$ the standard cone on $\Delta$, $\left(M_{\Delta}, \omega_{\Delta}, \Phi_{\Delta}: M_{\Delta} \rightarrow \mathfrak{g}^{*}\right)$ the compact connected integral symplectic toric manifold with moment polytope $\Delta$ and $\left(M_{C}, \omega_{C}, \Phi_{C}: M_{C} \rightarrow \mathfrak{g}^{*} \times \mathbb{R}^{*}\right)$ the symplectic toric cone corresponding to the cone $C$. Let $X$ denote the Liouville vector field on the symplectic cone $\left(M_{C}, \omega_{C}\right)$. Then

1. $S^{1}=\{1\} \times \mathbb{R} / \mathbb{Z} \subset G \times \mathbb{R} / \mathbb{Z}$ acts freely on $P_{C}:=\Phi_{C}^{-1}\left(\mathfrak{g}^{*} \times\left\{1^{*}\right\}\right)$ making it a principal $S^{1}$ bundle over $P_{C} / S^{1}$.

2. The quotient $P_{C} / S^{1}$ is naturally a symplectic toric $G$-manifold (isomorphic to) $\left(M_{\Delta}, \omega_{\Delta}, \Phi_{\Delta}\right)$.

3. $\alpha_{C}:=\left.\left(\iota(X) \omega_{C}\right)\right|_{P_{C}}$ is a connection 1-form on the principal $S^{1}$-bundle $\pi_{C}: P_{C} \rightarrow M_{\Delta}$.

4. $\pi_{C}{ }^{*} \omega_{\Delta}=d \alpha_{C}$.

Hence $\left(P_{C}, \alpha_{C}\right)$ is the Boothby-Wang manifold of $\left(M_{\Delta}, \omega_{\Delta}\right)$, and $\left(M_{C}, \omega_{C}\right)$ is the symplectization of $\left(P_{C}, \alpha_{C}\right)$.

Proof. The action of $S^{1}$ at a point $x \in M_{C}$ is free iff $S^{1}$ intersects trivially the isotropy group $H_{x}$ of $x$ in $H=G \times \mathbb{R} / \mathbb{Z}$. Since $M_{C}$ is $H$-toric, $H_{x}$ is connected hence a torus. Now two subtori $T_{1}, T_{2}$ of a torus $K$ intersect trivially iff their integral lattices $\mathbb{Z}_{T_{1}}, \mathbb{Z}_{T_{2}}$ intersect trivially and $\mathbb{Z}_{T_{1}}+\mathbb{Z}_{T_{2}}=\mathbb{Z}_{T_{1}} \oplus \mathbb{Z}_{T_{2}}$ is a direct summand of the integral lattice $\mathbb{Z}_{K}$ of $K$. The Lie algebra $\mathfrak{h}_{x}$ of $H_{x}$ is the annihilator of the face $F$ of $C$ containing $\Phi_{C}(x)$ in its interior. The integral lattice of $H_{x}$ is generated by the normals to the facets of $C$ intersecting in $F$.

Let us consider the worst case scenario: $\operatorname{dim} H_{x}=\operatorname{dim} H-1$, i.e., the face $F$ is the ray $\mathbb{R}^{>0}\left(v^{*}, 1^{*}\right)$ through a vertex $\left(v^{*}, 1^{*}\right)$ of $\Delta \times\left\{1^{*}\right\}$. The general case then follows easily from this special case. If $\left\{u_{i}^{*}\right\}$ is a basis of $\mathbb{Z}_{G}^{*}$ spanning the edges of $\Delta$ coming out of the vertex $v^{*}$, then the dual basis $\left\{u_{i}\right\} \subset \mathbb{Z}_{G}$ consists of the normals to the facets of $\Delta$ meeting at $v^{*}$. The vectors $\left\{\left(u_{i},-\left\langle v^{*}, u_{i}\right\rangle 1\right)\right\} \subset$ $\mathbb{Z}_{G} \times \mathbb{Z}=\mathbb{Z}_{H}$ are then the normal vectors to the facets of $C$ that intersect in the ray $\mathbb{R}^{>0}\left(v^{*}, 1^{*}\right)$. Clearly the set $\left\{\left(u_{i},-\left\langle v^{*}, u_{i}\right\rangle 1\right)\right\} \cup\{(0,1)\}$ is a basis of $\mathbb{Z}_{G} \times Z$. We conclude that the action of the $S^{1}$ on $M_{C}$ is free.

Since $S^{1}$ acts freely, 1 is a regular value of $f:=\left\langle\Phi_{C},(0,1)\right\rangle$. Hence $P_{C}=$ $f^{-1}(1)=\Phi_{C}^{-1}\left(\mathfrak{g}^{*} \times\left\{1^{*}\right\}\right)=\Phi_{C}^{-1}\left(\left(\mathfrak{g}^{*} \times\left\{1^{*}\right\}\right) \cap C\right)=\Phi_{C}^{-1}\left(\Delta \times\left\{1^{*}\right\}\right)$ is a principal $S^{1}$ bundle over $P_{C} / S^{1}$. It is connected since the fibers of $\Phi_{C}$ are connected. By the symplectic reduction theorem of Marsden - Weinstein and Meyer, $P_{C} / S^{1}$ is naturally a symplectic manifold. Moreover, since the actions of $S^{1}$ and $G$ 
commute, the action of $G$ on $M_{C}$ induces a Hamiltonian action of $G$ on $P_{C} / S^{1}$. Furthermore the restriction of $\Phi_{C}$ to $P_{C}$ descends to a corresponding $G$ moment map $\Phi$ on $P_{C} / S^{1}$ (provided we identify $\mathfrak{g}^{*}$ and $\mathfrak{g}^{*} \times\left\{1^{*}\right\}$ ). Clearly $\Phi\left(P_{C} / S^{1}\right)=$ $\Delta \times\left\{1^{*}\right\}$. Therefore by the uniqueness part of Delzant's classification of compact symplectic toric manifolds (Theorem 3.6 above) $P_{C} / S^{1}$ is $\left(M_{\Delta}, \omega_{\Delta}, \Phi_{\Delta}\right)$.

Finally we argue that $\omega_{\Delta}$ is the curvature of a connection 1-form on the principal $S^{1}$-bundle $\pi_{C}: P_{C} \rightarrow M_{\Delta}$. By construction $\pi_{C}{ }^{*} \omega_{\Delta}=\left.\omega_{C}\right|_{P_{C}}$. Let $Y$ denote the vector field on $M_{C}$ generating the action of the $S^{1}$. Then $\left\langle\Phi_{C},(0,1)\right\rangle=$ $\iota(Y) \iota(X) \omega_{C}$ (cf. equation (2.1)). Hence $1=\iota(Y) \alpha_{C}$ where $\left.\alpha_{C}:=\iota(X) \omega_{C}\right)\left.\right|_{P_{C}}$. It follows that $\alpha_{C}$ is a connection on $\pi_{C}: P_{C} \rightarrow M_{\Delta}$. Moreover, since $d \iota(X) \omega_{C}=$ $\omega_{C}$, we have $d \alpha_{C}=\left.\left(d \iota(X) \omega_{C}\right)\right|_{P_{C}}=\left.\omega_{C}\right|_{P_{C}}=\pi_{C}{ }^{*} \omega_{\Delta}$.

Next we apply Lemma 3.8 to construct certain contact toric 5-manifolds out of symplectic toric Hirzebruch surfaces. It will be convenient to take the standard $n$-torus $\mathbb{T}^{n}$ to be $\mathbb{R}^{n} / \mathbb{Z}^{n}$ and to identify the Lie algebra of $\mathbb{T}^{n}$ with $\mathbb{R}^{n}$. By using the standard basis of $\mathbb{R}^{n}$ we identify the dual of the Lie algebra of $\mathbb{T}^{n}$ with $\mathbb{R}^{n}$ and the weight lattice of $\mathbb{T}^{n}$ with $\mathbb{Z}^{n}$.

Definition 3.9. Following Karshon $[\mathrm{K}]$ we define the standard Hirzebruch trapezoid $\Delta(a, b, m)$ to be the quadrilateral in $\mathbb{R}^{2}$ with vertices $(0,0),(0, b),\left(a-\frac{m}{2} b, b\right)$ and $\left(a+\frac{m}{2} b, 0\right)$ where $m$ is a non-negative integer and $b>0, a>\frac{m}{2} b$ are real numbers.

By Theorem 3.6 there exists a symplectic 4-manifold $\left(M(a, b, m), \omega_{a, b, m}\right)$ with an effective Hamiltonian action of $\mathbb{T}^{2}$ such that $\Delta(a, b, m)$ is the image of $M(a, b, m)$ under the corresponding moment map. One can show that the manifold $M(a, b, m)$ is a Hirzebruch surface. In particular it is diffeomorphic to either $S^{2} \times S^{2}$ or to $\widetilde{\mathbb{C} P^{2}}$ ( $P^{2}$ with one point blown up), depending on the values of $a, b$, and $m$.

Definition 3.10. We define the standard Hirzebruch cone $C(a, b, m)$ to be the standard cone on the Hirzebruch trapezoid $\Delta(a, b, m)$ :

$$
C(a, b, m)=\left\{t\left(x_{1}, x_{2}, 1\right) \in \mathbb{R}^{3} \mid t \geq 0,\left(x_{1}, x_{2}\right) \in \Delta(a, b, m)\right\} .
$$

Now suppose that $b$ and $a-\frac{m}{2} b$ are integers. Then the Hirzebruch trapezoid $\Delta(a, b, m)$ is integral. It follows from Lemma 3.8 that $C(a, b, m)$ is the moment cone of the Boothby-Wang manifold $\left(P(a, b, m)\right.$, ker $\left.A_{a, b, m}\right)$ of the integral symplectic manifold $\left(M(a, b, m), \omega_{a, b, m}\right)$.

Proposition 3.11. Let $C(a, b, m)$ and $C\left(a^{\prime}, b^{\prime}, m^{\prime}\right)$ be two standard Hirzebruch cones with $b, b^{\prime}, a-\frac{m}{2} b, a^{\prime}-\frac{m^{\prime}}{2} b^{\prime} \in \mathbb{Z}$. If there is $T \in G L(3, \mathbb{Z})$ with $T(C(a, b, m))=C\left(a^{\prime}, b^{\prime}, m^{\prime}\right)$ then either $(a, b, m)=\left(a^{\prime}, b^{\prime}, m^{\prime}\right)$ or $m=m^{\prime}=0$ and $a=b^{\prime}, b=a^{\prime}$.

Proof. A Hirzebruch trapezoid $\Delta(a, b, m)$ has the following property: if $v_{0}$ is a vertex of $\Delta(a, b, m)$ and $v_{1}, v_{2}$ are two adjacent vertices then there is a basis $\left\{u_{1}, u_{2}\right\}$ of $\mathbb{Z}^{2}$ such that $v_{1}-v_{0}=t_{1} u_{1}, v_{2}-v_{0}=t_{2} u_{2}$ for some $t_{1}, t_{2}>0$. 
Depending on which three vertices we picked the set $\left\{t_{1}, t_{2}\right\}$ is either $\left\{b, a-\frac{m}{2} b\right\}$ or $\left\{b, a+\frac{m}{2} b\right\}$.

If $w \in \mathbb{Z}^{3}$ is a primitive vector then for any $T \in \mathrm{GL}(3, \mathbb{Z})$ the vector $T w$ is also primitive. Now suppose $T(C(a, b, m))=C\left(a^{\prime}, b^{\prime}, m^{\prime}\right)$. Then $T$ maps the edge of $C(a, b, m)$ through $(0,0,1)$ to an edge of $C\left(a^{\prime}, b^{\prime}, m^{\prime}\right)$ say through a vertex $v_{0}$ of $\Delta\left(a^{\prime}, b^{\prime}, m^{\prime}\right) \times\{1\} \subset \mathbb{R}^{3}$. Since both $T(0,0,1)$ and $v_{0}$ are primitive, we have $T(0,0,1)=v_{0}$. Similarly $T$ maps the vectors $(0, b, 1)$ and $\left(a+\frac{m}{2} b, 0,1\right)$ to vertices $v_{2}, v_{1}$ of $\Delta\left(a^{\prime}, b^{\prime}, m^{\prime}\right) \times\{1\}$ adjacent to $v_{0}$. It follows from the remark at the start of the proof that there are vectors $u_{1}, u_{2} \in \mathbb{Z}^{3}$ such that $\left\{v_{0}, u_{1}, u_{2}\right\}$ is a basis of $\mathbb{Z}^{3}$ and $v_{1}-v_{0}=t_{1} u_{1}, v_{2}-v_{1}=t_{2} u_{2}$ where

$$
\left\{t_{1}, t_{2}\right\}=\left\{b^{\prime}, a^{\prime}-\frac{m^{\prime}}{2} b^{\prime}\right\} \text { or }\left\{b^{\prime}, a^{\prime}+\frac{m^{\prime}}{2} b^{\prime}\right\} \text {. }
$$

Now $T(0,1,0)=T\left(\frac{1}{b}((0, b, 1)-(0,0,1))\right)=\frac{1}{b}\left(v_{2}-v_{0}\right)=\frac{t_{2}}{b} u_{2}$. Similarly $T(1,0,0)=\frac{t_{2}}{a+\frac{m}{2} b} u_{1}$. Since both $(0,1,0)$ and $u_{2}$ are primitive in $\mathbb{Z}^{3}, \frac{t_{2}}{b}= \pm 1$. Since $t_{2}>0$ we get

$$
t_{2}=b
$$

By the same argument

$$
t_{1}=a+\frac{m}{2} b .
$$

Finally let $v_{3}$ denote the remaining vertex of $\Delta\left(a^{\prime}, b^{\prime}, m^{\prime}\right) \times\{1\}$. Then $v_{3}=$ $T\left(a-\frac{m}{2} b, b, 1\right)=\left(a-\frac{m}{2} b\right) T(1,0,0)+b T(0,1,0)+T(0,0,1)$. Hence

$$
v_{3}=\left(a-\frac{m}{2} b\right) u_{1}+b u_{2}+v_{0} .
$$

Equation (3.2) gives us four cases to compare (3.5) with. For example suppose $t_{1}=a^{\prime}+\frac{m^{\prime}}{2} b^{\prime}$ and $t_{2}=b^{\prime}$. Then

$$
v_{3}=v_{0}+\left(a^{\prime}-\frac{m^{\prime}}{2} b^{\prime}\right) u_{1}+b^{\prime} u_{2}
$$

Comparing the above equation with equation (3.5) and using equations (3.3) and (3.4) we get:

$$
\begin{aligned}
a+\frac{m}{2} b & =a^{\prime}+\frac{m^{\prime}}{2} b^{\prime} \\
a-\frac{m}{2} b & =a^{\prime}-\frac{m^{\prime}}{2} b^{\prime} \\
b & =b^{\prime} .
\end{aligned}
$$

Hence $a=a^{\prime}, b=b^{\prime}, m=m^{\prime}$. The proposition follows by examining the remaining three cases.

Combing Proposition 3.11 with Remark 3.4 we see that the contact toric $\mathbb{T}^{3}$ manifolds $\left(P(a, b, m)\right.$, ker $\left.A_{a, b, m}\right)$ are isomorphic as contact toric manifolds if and only if either $(a, b, m)=\left(a^{\prime}, b^{\prime}, m^{\prime}\right)$ or $\left(m=m^{\prime}=0\right.$ and $\left.a=b^{\prime}, b=a^{\prime}\right)$. On the other hand, by Lemma 3 of $[\mathrm{K}]$ the manifold $\left(M(a, b, m), \omega_{a, b, m}\right)$ 
is symplectomorphic to $\left(M(a, b, m+2), \omega_{a, b, m+2}\right)$. Hence the 5 -manifolds $\left(P(a, b, m), \operatorname{ker} A_{a, b, m}\right)$ and $\left(P(a, b, m+2)\right.$, ker $\left.A_{a, b, m+2}\right)$ are contactomorphic. Now $P(a, b, m)$ is well-defined as long as $\frac{a}{b}>\frac{m}{2} \geq 0$. Let $k$ be the largest positive integer with $\frac{a}{b}>\frac{k}{2}$. Then $P(a, b, k), P(a, b, k-2), \ldots$ are contact toric $\mathbb{T}^{3}$-manifolds which are isomorphic as contact manifolds but not as contact toric manifolds. Note that there are $\ell$ of these manifolds where $k=2 \ell-2$ or $2 \ell-1$. This finishes our proof of Theorem 3.1.

Remark 3.12. By a theorem of Hatakeyama $[\mathrm{H}]$ the Boothby-Wang manifold of an integral Kaehler manifold is Sasakian. Hence the manifolds $P(a, b, m)$ considered above are all Sasakian. In particular they are all $K$-contact. Therefore Theorem 3.1 gives a positive answer to Problem 3 in [Y1].

\section{Contact structures on $S^{2} \times S^{3}$}

In this section we examine the manifolds constructed in the course of the proof of Theorem 3.1 in more details. We identify precisely what some of these manifolds are: for $a$ and $b$ relatively prime the manifolds $P(a, b, 0)$ turn out to be $S^{2} \times S^{3}$. We also identify the contact structures we have constructed as complex vector bundles. We do not know whether these contact structures which happen to be isomorphic as vector bundles are in fact isomorphic as contact structures.

Proposition 4.1. Suppose $a, b$ are two relatively prime integers with $a>b \geq 1$. Let $\left(P(a, b, 0), \xi_{a, b}=\operatorname{ker} A_{a, b, 0}\right)$ denote the corresponding contact toric 5manifold defined above. It is the Boothby-Wang manifold of $\left(\mathbb{C} P^{1} \times \mathbb{C} P^{1}, \omega_{a, b}\right)$.

1. $P(a, b, 0)$ is diffeomorphic to $S^{2} \times S^{3}$.

2. As a complex vector bundle $\xi_{a, b}$ is uniquely determined by the difference $a-b$.

Proof. The first part of the proposition is an observation of Wang and Ziller [WZ, (2.3)]. Since the manifold $P(a, b, 0)$ is the Boothby-Wang manifold of $\left(\mathbb{C} P^{1} \times \mathbb{C} P^{1}, \omega_{a, b}\right)$ the orbit map $\pi: P(a, b, 0) \rightarrow \mathbb{C} P^{1} \times \mathbb{C} P^{1}$ is a principal circle bundle with Chern class $\left[\omega_{a, b}\right]$. Principal torus bundles over products of KaehlerEinstein manifolds were studied extensively by Wang and Ziller, who showed that these bundles carry Einstein metrics (in the notation of [WZ] $P(a, b, 0)$ is $\left.M_{a, b}^{1,1}\right)$. In particular they showed that if $a$ and $b$ are relatively prime then $P(a, b, 0)$ is diffeomorphic to $S^{2} \times S^{3}$ (cf. [WZ, (2.3)]). ${ }^{2}$

We now prove part 2 of the theorem. Suppose $\left(a^{\prime}, b^{\prime}\right)$ is another pair of relatively prime integers with $a^{\prime}-b^{\prime}=a-b$. We claim that $\xi_{a, b}$ and $\xi_{a^{\prime}, b^{\prime}}$ are isomorphic as complex vector bundles. In the course of the proof of [WZ, (2.3)] Wang and Ziller showed that if one denotes the generator of $H^{2}(P(a, b, 0), \mathbb{Z})$ by $z$ and the obvious generators of $H^{2}\left(\mathbb{C} P^{1} \times\{*\}, \mathbb{Z}\right)$ and $H^{2}\left(\{*\} \times \mathbb{C} P^{1}, \mathbb{Z}\right)$ by $x$ and $y$ respectively, then $\pi^{*} x=a z$ and $\pi^{*} y=-b z$. Consequently the first Chern

\footnotetext{
${ }^{2}$ The rough idea of the proof is to first show that $P(a, b, 0)$ is simply connected and spin. It is easy to compute that $H^{2}(P(a, b, 0), \mathbb{Z})$ is $\mathbb{Z}$. Then Smale's classification of 5-dimensional simply connected spin manifolds implies that $P(a, b, 0)=S^{2} \times S^{3}$.
} 
class of the line bundle $L_{1}^{a, b}:=\pi^{*} T\left(\mathbb{C} P^{1} \times\{*\}\right)$ is $\pi^{*}(2 x)=2 a z$, and similarly the first Chern class of $L_{2}^{a, b}:=\pi^{*} T\left(\{*\} \times \mathbb{C} P^{1}\right)$ is $-2 b z$. Since the contact distribution $\xi_{a, b}$ is a connection on the bundle $\pi: P(a, b, 0) \rightarrow \mathbb{C} P^{1} \times \mathbb{C} P^{1}$, we have $\xi_{a, b}=\pi^{*} T\left(\mathbb{C} P^{1} \times \mathbb{C} P^{1}\right)$. Hence $\xi_{a, b}=L_{1}^{a, b} \oplus L_{2}^{a, b}$. The distribution $\xi_{a^{\prime}, b^{\prime}}$ is also a direct sum of line bundles: $\xi_{a^{\prime}, b^{\prime}}=L_{1}^{a^{\prime}, b^{\prime}} \oplus L_{2}^{a^{\prime}, b^{\prime}}$; additionally $c_{1}\left(L_{1}^{a^{\prime}, b^{\prime}}\right)=2 a^{\prime} z$ while $c_{1}\left(L_{2}^{a^{\prime}, b^{\prime}}\right)=-2 b^{\prime} z$. The projection $p: S^{2} \times S^{3} \rightarrow S^{2}$ induces an isomorphism on the level of second cohomology. Therefore all the line bundles over $P(a, b, 0)=S^{2} \times S^{3}=P\left(a^{\prime}, b^{\prime}, 0\right)$ are pull-backs by $p$ of line bundles over $S^{2}$, and consequently $\xi_{a, b}$ and $\xi_{a^{\prime}, b^{\prime}}$ are pull-backs by $p$ of rank 2 vector bundles over $S^{2}$. Two complex rank $n$ vector bundles over $S^{2}$ are isomorphic if and only if their determinant bundles are isomorphic. It follows that $\xi_{a, b}$ is isomorphic to $\xi_{a^{\prime}, b^{\prime}}$ iff $a-b=a^{\prime}-b^{\prime}$.

We end the paper with two questions.

Question 1. Suppose $a, b$ and $a^{\prime}, b^{\prime}$ are two different pairs of relatively prime integers with $a>b \geq 1, a^{\prime}>b^{\prime} \geq 1$ and $a-b=a^{\prime}-b^{\prime}$. Are the two contact manifolds $\left(P(a, b, 0), \xi_{a, b}\right)$ and $\left.P\left(a^{\prime}, b^{\prime}, 0\right), \xi_{a^{\prime}, b^{\prime}}\right)$ contactomorphic?

Question 2. What does Theorem 3.1 tell us about the topology of the group of contactomorphisms $\operatorname{Diff}\left(P(a, b, m)\right.$, ker $\left.A_{a, b, m}\right)$ ? For example, do the nonconjugate tori define different homology classes?

\section{Acknowledgments}

I am grateful to Sue Tolman and Charles Boyer for a number of useful conversations. Yael Karshon kindly provided me with a copy of her preprint. I thank Matthew Ando for answering several of my topology questions.

\section{References}

[Ab] M. Abreu, Topology of symplectomorphism groups of $S^{2} \times S^{2}$, Invent. Math. 131 (1998), 1-23.

[AbMc] M. Abreu, D. McDuff, Topology of symplectomorphism groups of rational ruled surfaces, J. Amer. Math. Soc. 13 (2000), 971-1009.

[BW] W. M. Boothby, H. C. Wang, On contact manifolds, Ann. of Math. (2) 68 (1958), 721-734.

[BG] C. P. Boyer, K. Galicki, On Sasakian-Einstein geometry, Internat. J. Math. 11 (2000), 873-909.

[DJ] M. W. Davis, T. Januszkiewicz, Convex polytopes, Coxeter orbifolds and torus actions, Duke Math. J. 62 (1991), 417-451.

[D] T. Delzant, Hamiltoniens périodiques et images convexes de l'application moment, Bull. Soc. Math. France 116 (1988), 315-339.

[G] M. Gromov, Pseudoholomorphic curves in symplectic manifolds, Invent. Math. 82 (1985), 307-347.

[H] Y. Hatakeyama, Some notes on differentiable manifolds with almost contact structures, Osaka Math. J. (2) 15 (1963), 176-181.

[K] Y. Karshon, Maximal tori in the symplectomorphism groups of Hirzebruch surfaces, Math. Res. Lett. 10 (2003), 125-132. 
[L2] E. Lerman, Contact toric manifolds, preprint: http://xxx.lanl.gov/abs/math.SG/0107201

[L3] — Contact cuts, Israel J. Math 124 (2001), 77-92.

[LS] E. Lerman, N. Shirokova, Completely integrable torus actions on symplectic cones, Math. Res. Lett. 9 (2002), 105-115.

[WZ] M. Y. Wang, W. Ziller, Einstein metrics on principal torus bundles, J. Differential Geom. 31 (1990), 215-248.

[Y1] T. Yamazaki, A construction of $K$-contact manifolds by fiber join, Tohoku Math. J. 51 (1999), 433-446.

Department of Mathematics, University of Illinois, Urbana, IL 61801, U.S.A.

E-mail address: lerman@math.uiuc.edu 